Nahar, Shamsun; Khan, Ruhul A.; Dey, Kamol; Sarker, Bapi; Das, Anjan K.; Ghoshal, Sushanta:

Comparative studies of mechanical and interfacial properties between jute and bamboo fiber-reinforced polypropylene-based composites

URN: $\quad$ urn:nbn:de:gbv:ilm1-2014210190

Published OpenAccess: October 2014

Original published in:

Journal of thermoplastic composite materials. - Thousand Oaks, Calif : Sage (ISSN 1530-7980). - 25 (2012) 1, S. 15-32.

DOI: $\quad 10.1177 / 0892705711404725$

URL: $\quad$ http://dx.doi.org/10.1177/0892705711404725

[Visited: 2014-10-14]

„Im Rahmen der hochschulweiten Open-Access-Strategie für die Zweitveröffentlichung identifiziert durch die Universitätsbibliothek IImenau."

"Within the academic Open Access Strategy identified for deposition by Ilmenau University Library."

"Dieser Beitrag ist mit Zustimmung des Rechteinhabers aufgrund einer (DFG-geförderten) Allianz- bzw. Nationallizenz frei zugänglich."

"This publication is with permission of the rights owner freely accessible due to an Alliance licence and a national licence (funded by the DFG, German Research Foundation) respectively."

\section{DFG}

Nationallizenzen 


\section{Journal of Thermoplastic Composite Materials \\ http://jtc.sagepub.com/}

Comparative Studies of Mechanical and Interfacial Properties between Jute and Bamboo Fiber-Reinforced Polypropylene-Based Composites

S. Nahar, R.A. Khan, K. Dey, B. Sarker, A.K. Das and S. Ghoshal Journal of Thermoplastic Composite Materials 2012 25: 15 originally published online 21 July 2011

DOI: $10.1177 / 0892705711404725$

The online version of this article can be found at:

http://jtc.sagepub.com/content/25/1/15

Published by:

(S)SAG

http://www.sagepublications.com

Additional services and information for Journal of Thermoplastic Composite Materials can be found at:

Email Alerts: http:/jtc.sagepub.com/cgi/alerts

Subscriptions: http://jtc.sagepub.com/subscriptions

Reprints: http://www.sagepub.com/journalsReprints.nav

Permissions: http://www.sagepub.com/journalsPermissions.nav

Citations: http://jtc.sagepub.com/content/25/1/15.refs.html

>> Version of Record - Feb 15, 2012

OnlineFirst Version of Record - Jul 21, 2011

What is This? 


\title{
Comparative Studies of Mechanical and Interfacial Properties between Jute and Bamboo Fiber-Reinforced Polypropylene-Based Composites
}

\author{
Shamsun Nahar, ${ }^{1,2}$ Ruhul A. Khan, ${ }^{1, *}$ Kamol Dey, ${ }^{1,3}$ Bapi Sarker, ${ }^{3}$ \\ Anjan K. Das ${ }^{3}$ and Sushanta Ghoshal ${ }^{3,4}$ \\ ${ }^{1}$ Nuclear and Radiation Chemistry Division, Institute of Nuclear Science and \\ Technology, Bangladesh Atomic Energy Commission, Dhaka 1000, \\ Bangladesh \\ ${ }^{2}$ Department of Chemistry, Bangladesh College of Leather Engineering and \\ Technology, Dhaka, Bangladesh \\ ${ }^{3}$ Department of Applied Chemistry \& Chemical Engineering, Faculty of \\ Engineering and Technology, University of Dhaka, Dhaka 1000, Bangladesh \\ ${ }^{4}$ Department of Technical Physics II, Faculty of Mathematics and Natural \\ Science, Ilmenau University of Technology, Ilmenau, Germany
}

\begin{abstract}
Jute and bamboo fiber-reinforced polypropylene (PP) based composites ( $50 \mathrm{wt} \%$ fiber) were fabricated by compression molding. Tensile strength (TS), bending strength (BS), tensile modulus (TM), and bending modulus (BM) of the jutereinforced PP composite were found to be 48, 56, 900, and $1500 \mathrm{MPa}$, respectively. Then, bamboo fiber-reinforced PP-based composites ( $50 \mathrm{wt} \%$ fiber) were fabricated and the mechanical properties evaluated. The TS, BS, TM, and BM of bambooreinforced PP composites were found to be $60,76,4210$, and $6210 \mathrm{MPa}$, respectively. It was revealed that bamboo fiber-based composites had higher TS, BS, TM, and BM compared to jute-based composites. Degradation tests of the composites (jute fiber/PP and bamboo fiber/PP) were performed in soil at ambient conditions for up to 24 weeks. It was revealed that bamboo fiber/PP composite retained its original mechanical properties higher than that of jute fiber/PP composite.
\end{abstract}

\footnotetext{
*Author to whom correspondence should be addressed.

E-mail: dr.ruhul_khan@yahoo.com

Figures 1-6 appear in color online http://jtc.sagepub.com
}

Journal of Thermoplastic COMPOSite Materials, Vol. 25-February 2012 
The interfacial shear strength of the jute and bamboo fiber-based composites was investigated using the single-fiber fragmentation test and it was found to be 2.14 and $4.91 \mathrm{MPa}$, respectively. Fracture sides of the composites were studied by scanning electron microscope, and the results revealed poor fiber matrix adhesion for jute fiber-based composites compared to that of the bamboo fiber-based composites.

KEY WORDS: composites, polypropylenejute fabrics, bamboo fibers, interfacial properties.

\section{INTRODUCTION}

$\mathbf{F}$ OR A FEW decades, efforts are going on to utilize natural fibers due to their biodegradability, availability, and low cost. Moreover, ecological concerns have initiated a considerable interest in natural materials to produce 'green' products. The rapidly increasing environmental awareness, growing global waste problem, limited availability of crude oils, and high processing cost trigger the development concepts of sustainability and reconsideration of renewable resources. Studies on composites have also been done concerning natural fiber-reinforced structures. Natural fibers have already established a record of accomplishment as reinforcing material. The advantages of natural fiber-reinforced composites are reduction in density of products, acceptable specific strength, toughness, and stiffness in comparison with synthetic fiber-reinforced composites, lower energy consumption from fiber growing to finished composites, the relative safety in the case of manufacturing processes when compared with synthetic-based reinforced composites, and low capital investment due to low cost of natural fibers compared to synthetic ones [1,2]. For this, natural fibers such as jute, flux, hemp, bamboo, etc. can be alternately used to reduce the cost of the composites. Jute has high specific properties like low density, less abrasive behavior to the processing equipment, good dimensional stability, and harmlessness. Jute textile products are low-cost eco-friendly products, abundantly available, easy to transport, and have superior drapability and moisture retention capacity. They are widely being used as a natural choice for plant mulching and rural road pavement construction. The biodegradable and low-priced jute products merge with the soil after providing nourishment to the soil. Being made of cellulose, on combustion, jute does not generate toxic gases $[3,4]$. There are several reports about the use of jute as reinforcing fibers for thermo-sets and thermoplastics. The studies of jute fiber composites were carried out mostly in the Indian 
subcontinent in the early years and jute fiber-reinforced thermo-sets had been the focus of studies. Fiber treatment and interfacial modification were the most frequently studied issues aiming to improve the properties of short or long fiber-reinforced composites [5-9]. Bamboo is a natural fiber traditionally used to create various living facilities and tools; the high strength value to its weight is derived from fibers longitudinally aligned in its body. Bamboo fibers are generally called 'natural glass fibers' even though they have not been studied as much as glass fibers. Bamboo is a naturally occurring composite material which grows abundantly in most of the tropical countries. It is considered a composite material because it consists of cellulose fibers imbedded in a lignin matrix. Cellulose fibers are aligned along the length of the bamboo providing maximum tensile flexural strength and rigidity in that direction [10]. It has been found that bamboo fiber bundles have a potential ability to work as the reinforcement of polymer matrix and show higher tensile strength (TS) than jute fibers and improved the bio-composites from biodegradable polymers [11]. Bamboo fiber, which is a cellulose fiber, can be easily blended with the other natural fibers as well as it is recyclable under $100 \%$ sun light or at the soil with the microorganisms; therefore, it is known as a 'natural green and economic fiber' and a 21 st century material which also has antibacterial properties. Therefore, the bamboo fiber-reinforced composite structures, with their mechanical properties, can benefit from the fiber's natural qualities and reasonable good physical properties [12].

Jute fiber contains $82-85 \%$ of holocellulose of which $58-63 \%$ is $\alpha$-cellulose. Jute fibers present some disadvantages such as high moisture sorption, poor dimensional stability, intrinsic polarity, low thermal resistance, anisotropic fiber resistance, and variability [4]. Bamboo fiber contains $68-75 \%$ of holocellulose, of which $45-49 \%$ is $\alpha$-cellulose. Cellulose is the main source of the mechanical properties of bamboo. In the structure of cellulose molecule in bamboo, three planes are defined; they are mutually perpendicular. The cellulose molecules are kept together in the first plane by numerous and hydrogen bonds, in the second plane by much weaker Van der Waals forces, and in the third plane by covalent bonds [12]. As a matrix material, polypropylene (PP) was used in this study because it has some excellent characters for composite fabrication. $\mathrm{PP}$ is a class of thermoplastic engineering polymer and it possesses several imperative and useful properties such as transparency, dimensional stability, flame resistance, high heat-distortion temperature, and high IS, and these widen its application. PP is also very suitable for filling, reinforcing, and blending. PP with fibrous natural fibers is one of the potential routes to create natural synthetic polymer composites $[13,14]$. 
In the case of polymer-fiber systems, the quality of the interface is very important as it controls the mechanical properties of the resulting composites. The interfacial properties of fiber-reinforced composites can be measured using the single-fiber fragmentation test (SFFT). The SFFT method has several advantages such as: simple specimen handling, replication of the stress-transfer characteristics in real composites, convenient monitoring of failure processes directly, and the fact that critical length is sensitive to the level of fiber-matrix adhesion. Interface scientists regard this test as a reliable method for the determination of interfacial properties of the fiber-reinforced polymer composites [15-23].

The mechanical properties of the matrix (PP) and the composites (jute fibers/PP and bamboo fibers/PP) were measured and jute fiber/PP composites compared over the bamboo fiber/PP composites. SFFT method was used to measure the interfacial properties of jute and bamboo fiber-based composites. The ultimate aim of these research studies was to compare the mechanical and interfacial properties between jute fiber/PP and bamboo fiber/PP composites.

\section{EXPERIMENTAL}

\section{Materials}

PP granules manufactured by Cosmoplene Polyolefin Company Ltd, Singapore, were used in as received condition in this experiment. Bleached jute fabrics were obtained from the Bangladesh Jute Research Institute (BJRI), Dhaka. Bamboo fiber was collected from Sylhet Division of Bangladesh.

\section{Water Uptake of the Jute and Bamboo Fibers}

Water uptake of jute and bamboo fiber (about $500 \mathrm{mg}$ ) was carried out in deionized water at room temperature $\left(25^{\circ} \mathrm{C}\right)$ for up to $60 \mathrm{~min}$. Both fiber samples were placed in static glass beakers containing $100 \mathrm{~mL}$ of deionized water. At set time points, samples were taken out and dried for $6 \mathrm{~h}$ at $105^{\circ} \mathrm{C}$ and then reweighed.

\section{Degradation Tests of Jute and Bamboo Fibers in Aqueous Medium}

Degradation tests of jute and bamboo fibers (about $500 \mathrm{mg}$ ) were performed in deionized water at room temperature $\left(25^{\circ} \mathrm{C}\right)$ up to 6 weeks. 
Samples (both jute and bamboo) were placed into static glass beakers containing $250 \mathrm{~mL}$ of deionized water. At set time points, samples were taken out, and dried for $6 \mathrm{~h}$ at $105^{\circ} \mathrm{C}$, and then reweighed. Similar degradation tests were reported in the literature [24-29].

\section{Fabrication of Unidirectional Composite}

Granules of PP (about $6 \mathrm{~g}$ ) were placed in two steel plates for making one PP sheet and then into the heat press (Carver, INC, USA Model 3856). Steel plates were pressed at 5 bar consolidation pressure for $1 \mathrm{~min}$ at $180^{\circ} \mathrm{C}$. The plates were then cooled for $1 \mathrm{~min}$ in a separate press under 5 bar pressure at room temperature. For composite fabrication, the resulting PP sheet was cut into desired size for composite fabrication. Unidirectional composites were prepared by sandwiching four layers of jute fiber between five sheets of PP. The sandwich was then placed between two steel plates and heated at $180^{\circ} \mathrm{C}$ for $5 \mathrm{~min}$ to soften the polymer prior to pressing 5 bar pressure for $5 \mathrm{~min}$. The fiber weight fraction for jute composites was calculated to be about 50\%. Unidirectional bamboo composite was also fabricated following the similar parameters in the same heat press. The fiber weight fraction for bamboo composite was calculated to be about $50 \%$.

\section{Mechanical Properties of the Composites}

The Hounsfield series S testing machine (UK) with a cross-head speed of $1 \mathrm{mms}^{-1}$ at a span distance of $25 \mathrm{~mm}$ was used to measure the tensile and bending properties of the composites. The dimensions of the test specimen were (ISO 14125): $60 \times 15 \times 2 \mathrm{~mm}^{3}$. A band saw was used to cut the composite samples to the required dimension. IS (Charpy) of the composites was measured using Impact tester (MT-3016, Pendulum type, Germany). Hardness was determined by HPE Shore-A Hardness Tester (model 60578, Germany).

\section{Soil Degradation Tests of the Composites}

Composite samples were buried in soil (having at least 25\% moisture) for different periods of time. After a certain period, samples were withdrawn carefully, washed with distilled water, and dried at $105^{\circ} \mathrm{C}$ for $6 \mathrm{~h}$, kept at 
room temperature $\left(25^{\circ} \mathrm{C}\right)$ for $24 \mathrm{~h}$, and then measured for their mechanical properties.

\section{Interfacial Properties of the Composite}

Single-fiber composite samples were prepared using a single filament of fiber (both jute and bamboo) between two sheets of PP. The sandwich was then hot pressed at $180^{\circ} \mathrm{C}$ for $1 \mathrm{~min}$ at 5 bar pressure between two steel plates. The plates were cooled in a separate press at 3 bar pressure to room temperature. The thickness of the specimen was $0.40 \mathrm{~mm}$. The single-fiber composite specimens $\left(25 \times 5 \times 0.40 \mathrm{~mm}^{3}\right)$ were loaded on the tensile machine (Hounsfield series $\mathrm{S}$ testing machine, UK) to bring out the repeated breakage of the fiber. A cross-head speed of $0.25 \mathrm{~mm} / \mathrm{min}$ was used. The gage length was $25 \mathrm{~mm}$. The experiment was monitored by a microscope (Hitachi) attached to a monitor. To reach the saturation level, the number of fragments over the $25-\mathrm{mm}$ gage length at each load level (using $2 \mathrm{~N}$ increments) was counted. Similarly, the saturation point was also checked by the number of fragments against displacement. The critical length $\left(l_{c}\right)$ was then measured using the formula: $l_{c}=4 l_{f} / 3$, where $l_{f}$ is the average fragment length which was calculated as the monitored length $(25 \mathrm{~mm})$ divided by the number of breaks observed within that length of the experimental fragment length distribution, according to Kelly-Tyson model [19-21]. To find out the critical length, the number of fragments was counted. Fiber tensile properties were obtained by tensile testing filaments using the international standard BS ISO 11566. A single fiber was mounted on a paper frame with a gage length of $25 \mathrm{~mm}$. The fiber was fastened to the frame with epoxy adhesive. Once prepared, the sample was gripped in the tensile machine. Before starting the test, the paper sections were cut. A cross-head speed of $1 \mathrm{~mm} / \mathrm{min}$ was used. The interfacial shear strength (IFSS) of the composites was calculated from both the Kelly-Tyson and Drzal equations [20-23]. Based on the force balance on a micro-mechanical model, Kelly and Tyson showed that IFSS is given by: $\Gamma_{i}=d \times \sigma_{f} / 2 l_{c}$, where $d$ is the fiber diameter, $\sigma_{f}$ the single-fiber TS at the critical fragment length $l_{c}$. Drzal et al. [20] altered the above equation to reflect Weibull statistics to form: $\Gamma_{i}=\sigma_{f} \Gamma / 2 \alpha[1-1 / \beta]$, where $\alpha$ and $\beta$ are the scale and shape parameters, respectively, in the Weibull distribution for the aspect ration and $\Gamma$ the gamma function. Fiber strength can be calculated from the extrapolation gage length using the Weibull weakest chart rule [20]. The fiber strength at the critical fragment length is: $\sigma_{f}=\sigma_{f 0}\left(l_{c} / l_{0}\right) \exp (1 / \beta)$, where $\sigma_{f 0}$ is the fiber strength at gage length $l_{0}$ and $\beta$ the shape parameter of the Weibull distribution for the fiber TS. 


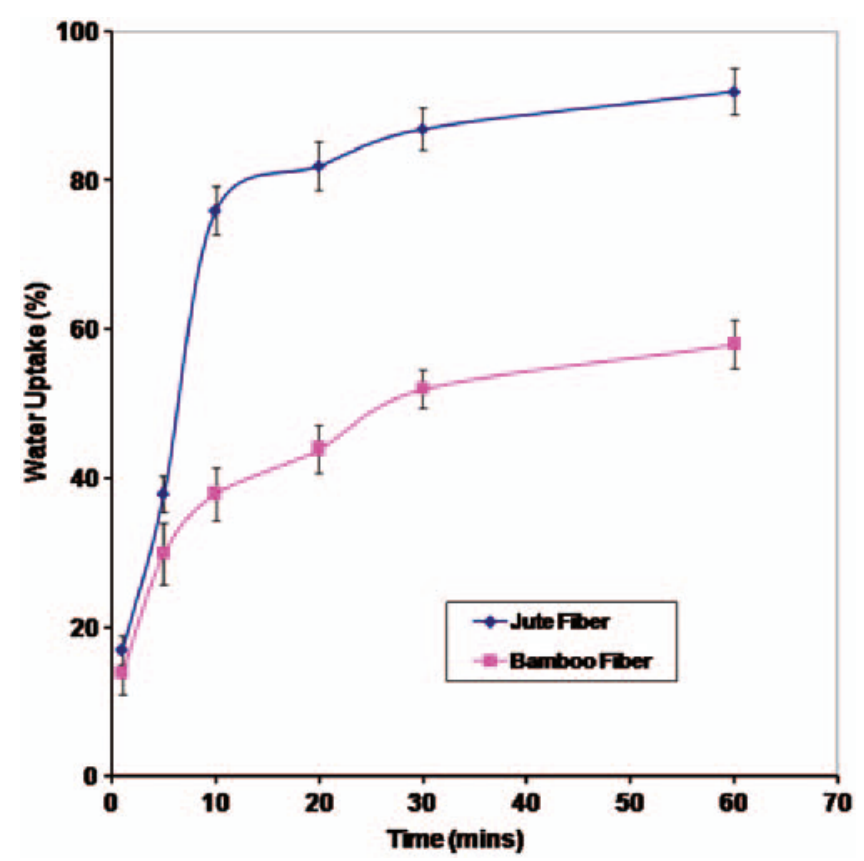

Figure 1. Percentages of water uptake (\%) by jute and bamboo fibers in aqueous media at room temperature $\left(25^{\circ} \mathrm{C}\right)$.

\section{Scanning Electron Microscopic Analysis}

Jute and bamboo fibers were examined by Phillips scanning electron microscope (SEM) at an accelerating voltage of $10 \mathrm{kV}$. SEM specimens were sputter-coated with gold. Fracture sides of the composites (after bending tests) were also observed using SEM.

\section{RESULTS AND DISCUSSION}

\section{Water Uptake of Jute and Bamboo Fibers}

Water uptake of jute and bamboo fibers was measured by soaking the fiber in a static glass beaker containing deionized water at room temperature $\left(25^{\circ} \mathrm{C}\right)$ for $60 \mathrm{~min}$. The results were presented in Figure 1. It was found that jute absorbs water in a typical manner, that is, gain of water in the initial stage was very rapid and then the absorption became slower and static 
with time. For example, after 1 min of immersion in water, jute absorbed $17 \%$ of water, but $76 \%$ and $92 \%$ of mass was gained after 10 and $60 \mathrm{~min}$, respectively. Basically, jute absorbed most of water within $10 \mathrm{~min}$ of immersion in water. On the other hand, bamboo fiber absorbed water less rapidly than the jute fiber and became static after $60 \mathrm{~min}$. For instance, bamboo fiber absorbed $14 \%$ water after 1 min immersion in water, but $38 \%$ and 58\% mass gain evidenced after 10 and $60 \mathrm{~min}$, respectively. After $60 \mathrm{~min}$, mass gain of bamboo and jute fibers reached $58 \%$ and $92 \%$, respectively. From this investigation, it was clear that, bamboo fiber absorbed water more slowly than the jute fiber. Jute is mainly built up of cellulose, which is a hydrophilic glucose polymer. The elementary unit of jute is anhydro-D-glucose, which contains three hydroxyl $(-\mathrm{OH})$ groups [4,17]. These hydroxyl groups in the cellulose structure account for the strong hydrophilic nature of jute and as a result, within an hour, jute absorbs such a huge amount of water [24]. Bamboo fiber contains 42-49\% cellulose, 26-27\% pentosan, 25-26\% lignin, 3-6\% ethanol toluene extractives, and $1-2 \%$ ashes. This higher amount of lignin in bamboo fiber makes it less hydrophilic than jute fiber [25,26], which is responsible for lower water uptake of bamboo fiber compare to the jute fiber.

\section{Degradation Tests of Jute and Bamboo Fibers in Aqueous Medium}

Six weeks of degradation (mass loss) of jute and bamboo fibers were performed in deionized water at room temperature $\left(25^{\circ} \mathrm{C}\right)$, and the results are depicted in Figure 2. It was found that jute and bamboo fibers degraded slowly in aqueous medium but jute fiber degraded more rapidly compared to the bamboo fiber. Jute lost $4 \%$ of mass after 1 week of immersion in water but $22 \%$ and $30 \%$ mass losses were found in 3 and 6 weeks, respectively. On the contrary, bamboo fiber lost $2.5 \%$ of mass after 1 week of immersion in water but $15 \%$ and $24 \%$ mass losses were found in 3 and 6 weeks, respectively. On the contrary, bamboo fiber lost $2.5 \%$ of mass after 1 week of immersion in water but $15 \%$ and $24 \%$ losses of mass were found in 3 and 6 weeks, respectively. Jute is composed of cellulose ( $\alpha$-cellulose and hemicellulose), binding materials (lignin, fats, and waxes), and minor constituents (inorganic and nitrogenous matters, and traces of pigments like $\beta$-carotene and xanthophylls) [24]. Leaching out of non-cellulosic materials occurs lost during initial hours; then fiber degradation occurs; and these are the causes of the loss of weight of jute fiber. This finding suggests that jute is a rapidly degraded natural fiber. The slow degradation of the bamboo fiber compared to the jute fiber in the aqueous medium was expected because of the less hydrophilic nature of bamboo fiber $[25,26]$. 


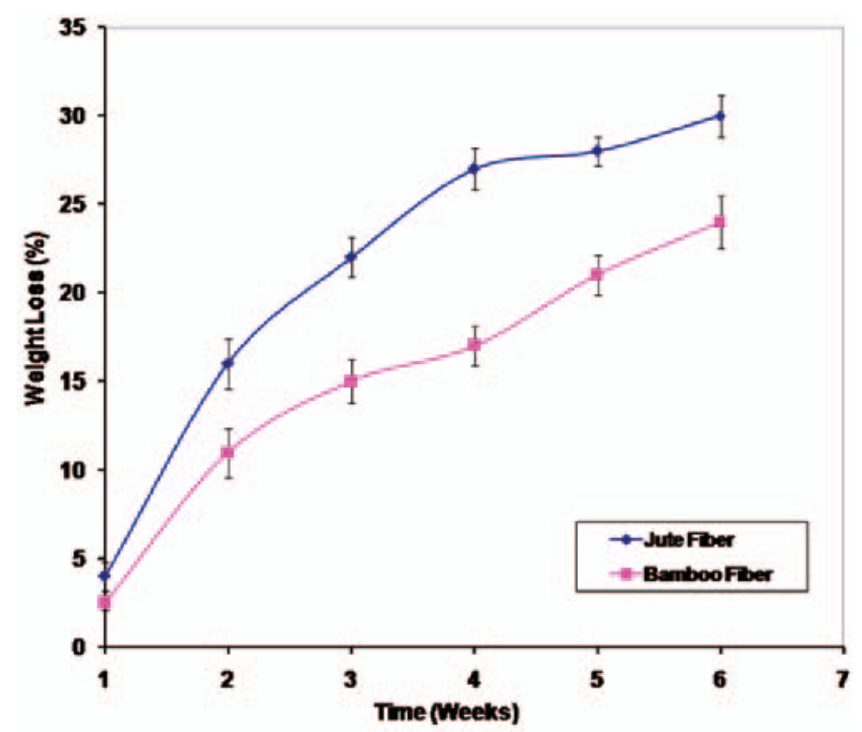

Figure 2. Degradation of jute and bamboo fibers in the aqueous medium at room temperature $\left(25^{\circ} \mathrm{C}\right)$.

\section{Comparative Studies of the Mechanical Properties of the Composites}

The mechanical properties such as tensile and bending properties of the PP sheet, jute fiber/PP, and bamboo fiber/PP composites were evaluated, and the values given in Tables 1 and 2. It was found that TS, tensile modulus (TM), elongation at break (\%), bending strength (BS), and bending modulus (BM) of the PP sheet were found to be $21 \mathrm{MPa}, 530 \mathrm{MPa}, 378 \%$, $27 \mathrm{MPa}$, and $835 \mathrm{MPa}$, respectively. Jute-based composites made of $50 \%$ fiber significantly improved the mechanical properties (TS, TM, BS, and $\mathrm{BM})$. TS, TM, BS, and BM for the jute fiber/PP composite were found 48 , 900 , 56, and $1500 \mathrm{MPa}$, respectively. Jute composites gained $128 \%$ increase in TS and 107\% increase in BS over that of the matrix PP. It was also found that TM and BM improved by $70 \%$ and $80 \%$, respectively than that of the matrix material PP. On the other hand, percentage elongation at break $(\mathrm{Eb} \%)$ was reduced drastically because of low $\mathrm{Eb} \%$ of the fibers compared to PP. From this investigation, it was clear that jute composites gained huge mechanical properties over the matrix material and thus indicated good fiber matrix adhesion.

From Table 2, it was very clear that bamboo composite possessed a significant improvement in TS, BS, TM, and BM compared to the matrix PP. TS, TM, BS, and BM of bamboo fiber/PP composite were found 60 , 
Table 1. Tensile properties of polypropylene (PP) sheet, jute fiber/PP, and bamboo fiber/PP composites (50\% fiber by weight).

\begin{tabular}{lccc}
\hline & \multicolumn{3}{c}{ Tensile properties } \\
\cline { 2 - 4 } Material & $\begin{array}{c}\text { Strength } \\
\text { (MPa) }\end{array}$ & $\begin{array}{c}\text { Modulus } \\
\text { (MPa) }\end{array}$ & $\begin{array}{c}\text { Elongation } \\
\text { at break (\%) }\end{array}$ \\
\hline PP sheet & $21 \pm 2$ & $530 \pm 120$ & $378 \pm 35$ \\
Jute fiber/PP composite & $48 \pm 3$ & $900 \pm 200$ & $12 \pm 3$ \\
Bamboo fiber/PP composite & $60 \pm 3$ & $4210 \pm 750$ & $4 \pm 1$ \\
\hline
\end{tabular}

Table 2. Bending properties of PP sheet, jute fiber/PP, and bamboo fiber/PP composites ( $50 \%$ fiber by weight).

\begin{tabular}{lcc}
\hline & \multicolumn{2}{c}{ Bending properties } \\
\cline { 2 - 3 } Material & $\begin{array}{c}\text { Strength } \\
(\mathrm{MPa})\end{array}$ & $\begin{array}{c}\text { Modulus } \\
\text { (MPa) }\end{array}$ \\
\hline PP sheet & $27 \pm 2$ & $835 \pm 150$ \\
Jute fiber/PP composite & $56 \pm 3$ & $1500 \pm 180$ \\
Bamboo fiber/PP composite & $76 \pm 4$ & $6210 \pm 450$ \\
\hline
\end{tabular}

4210,76 , and $6210 \mathrm{MPa}$, respectively. It was revealed that the bamboo fiber/ PP composites were found to have $185 \%$ and $180 \%$ improvements in TS and BS over the matrix PP. TM and BM also improved and they increased by $694 \%$ and $643 \%$, respectively, over the matrix material PP. The increased mechanical properties were attributed due to the reinforcement of the bamboo fiber with the matrix PP. Bamboo-based composites showed significantly higher TS, BS, TM, and BM over the jute composites. It was revealed that the bamboo fiber/PP composites were found to have $25 \%$ and $35 \%$ improvements of TS and BS over the jute composites. It was also found that TM and BM improved by $367 \%$ and $314 \%$, respectively, than that of the jute/PP composites. Bamboo is less hydrophilic than jute due to the higher percentage of lignin content in bamboo. As PP is hydrophobic in nature, better adhesion occurred between bamboo fiber and PP compared to the jute fiber; thereby, bamboo fiber/PP composite showed higher TS, BS, $\mathrm{TM}$, and $\mathrm{BM}$ over the jute fiber/PP composite.

\section{Comparative Studies of the Degradation Tests of the Composites in Aqueous Medium}

Degradation tests of the composites (jute fiber/PP and bamboo fiber/PP) were performed in soil at ambient conditions for up to 24 weeks. TS and BS values were plotted against degradation time as shown in Figures 3 and 4. 


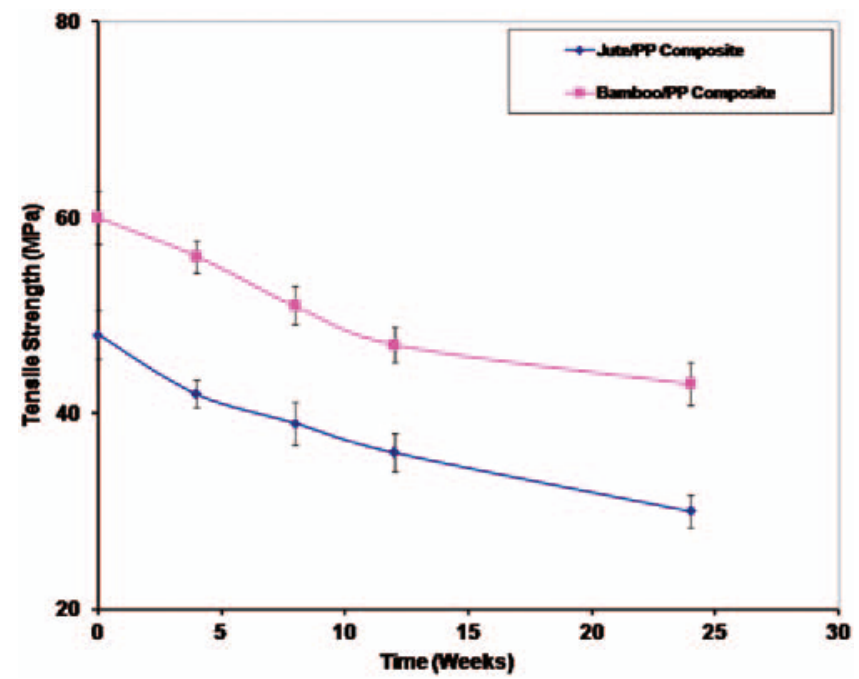

Figure 3. Degradation of TS of the composites during soil degradation tests.

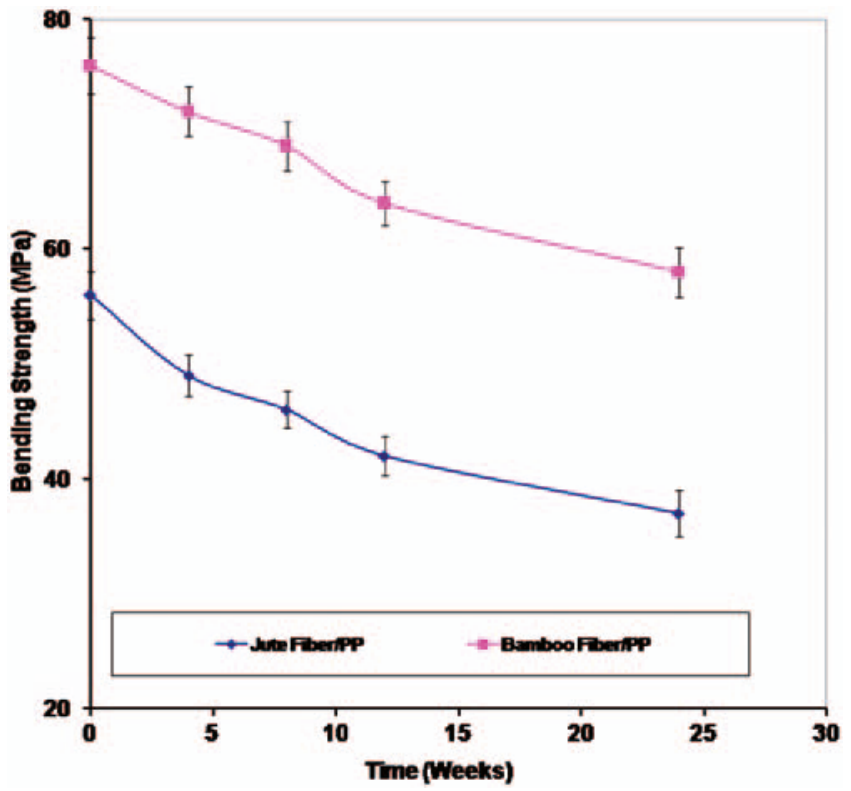

Figure 4. Degradation of BS of the composites during soil degradation tests. 
It was found that for jute/PP composites, both TS and BS decreased slowly with time but for bamboo fiber/PP, a very slow decrease was observed. After 24 weeks of soil degradation, jute composites lost almost $40 \%$ and $36 \%$ of TS and BS, respectively. On the other hand, bamboo fiber/PP composites lost $28 \%$ and $25 \%$ of TS and BS, respectively. Similarly, TM and BM also decreased over soil degradation time and the results were depicted in Figures 5 and 6 . It was found that, jute fiber/PP composites lost $46 \%$ and $35 \%$ of $\mathrm{TM}$ and $\mathrm{BM}$, respectively, during soil degradation test. After 24 weeks of soil degradation, bamboo fiber/PP composites lost almost $24 \%$ and $25 \%$ of $\mathrm{TM}$ and $\mathrm{BM}$, respectively. From this investigation, it was evident that bamboo fiber/PP composites retained much of their original mechanical properties than the jute composites during soil degradation. Jute is a natural biodegradable fiber and this fiber being cellulose based, it absorbs water within a couple of minutes indicating its strong hydrophilic character. Cellulose has a strong tendency to degrade when buried in soil [27]. During soil-degradation tests, water penetrated from the cutting edges of the composites in jute-based samples and degradation of cellulose occurred in jute; as a result, the mechanical properties of the composites decreased significantly. But bamboo fiber contains higher percentage of lignin and lower percentage of cellulose than jute and thus bamboo fiberbased composites are less hydrophilic in nature than jute-based composite. These higher percentages of lignin repelled water more significantly than the jute fiber. So, bamboo fiber/PP composite retained much of its original mechanical properties during exposure to the soil.

\section{Comparative Studies of the Interfacial Properties of the Composites}

To find out the IFSS, SFFT was carried out. Single filament (bamboo fiber and jute fiber) reinforced PP matrix composites were prepared by compression molding. Fragmentation test was performed using universal testing machine and the number of fragments was counted using microscope operated at transmission mode. The results were given in Table 3. The PP matrix that was quite transparent, which facilitated the counting of the number of fiber fragments in the specimen. For bamboo fiber/PP systems, the total number of fragments reached 8 and the critical length was found to be $3500 \mu \mathrm{m}$, calculated according to the equation mentioned in the 'Experimental' section. TS value of the fiber at the critical length was measured using the Weibull weakest chart rule and found to be $625 \mathrm{MPa}$. The diameter of the fiber used in this experiment varied from $55 \pm 3 \mu \mathrm{m}$. The IFSS was found to be $4.91 \mathrm{MPa}$ for bamboo fiber/PP system. This investigation revealed that the bamboo fiber/PP composite had 


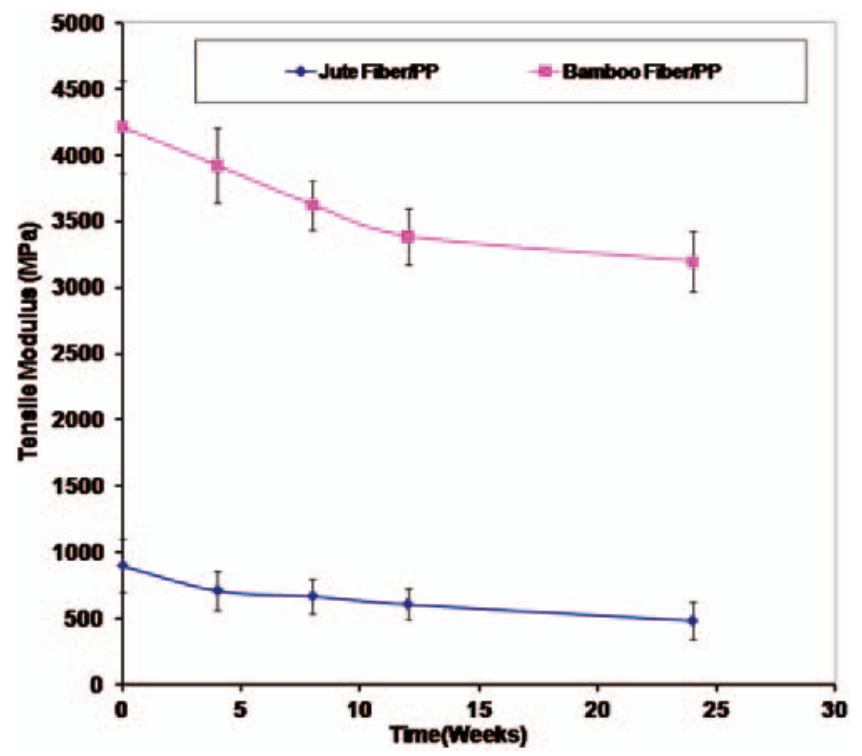

Figure 5. Degradation of TM of the composites during soil degradation tests.

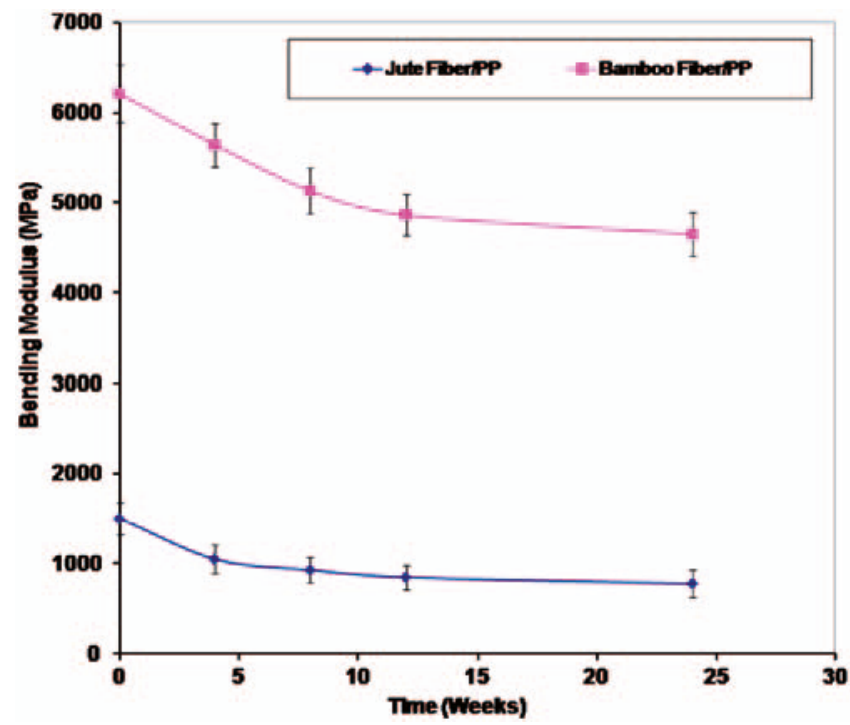

Figure 6. Degradation of $B M$ of the composites during soil degradation tests. 
Table 3. IFSS of jute fiber/PP and bamboo fiber/PP composites.

\begin{tabular}{lcccc}
\hline Composite system & $\begin{array}{c}\text { Critical fiber } \\
\text { length }(\mu \mathrm{m})\end{array}$ & $\begin{array}{c}\text { TS of fiber } \\
\text { at the critical } \\
\text { length }(\mathbf{M P a})\end{array}$ & $\begin{array}{c}\text { Fiber } \\
\text { diameter } \\
(\mu \mathrm{m})\end{array}$ & $\begin{array}{c}\text { IFSS } \\
(\mathbf{M P a})\end{array}$ \\
\hline Jute fiber/PP system & $3733 \pm 120$ & $455 \pm 12$ & $35 \pm 2$ & $2.14 \pm 0.2$ \\
Bamboo fiber/PP system & $3500 \pm 110$ & $625 \pm 14$ & $55 \pm 3$ & $4.91 \pm 0.3$ \\
\hline
\end{tabular}

sufficient IFSS. For the jute fiber/PP composite system, the number of fragments reached was 7 for the single-fiber composite sample and the critical length was found to be $3733 \mu \mathrm{m}$, which was lower than that of the bamboo fiber/PP system. The TS value of the jute fiber/PP system was found to be $455 \mu \mathrm{m}$. The IFSS value was found to be $2.14 \mathrm{MPa}$ for jute fiber/ PP system. The IFSS value of bamboo fiber/PP composite was higher compared to the jute fiber/PP composite. Jute is more hydrophilic than bamboo, but PP is hydrophobic in nature; as a result, the IFSS was found to be low for jute fiber/PP system compared to that of the bamboo fiber/PP system.

\section{SEM Images of the Composites}

To find out the fiber matrix adhesion inside the composites, SEM studies were carried out. SEM images of jute fiber (a) and the fracture sides of the corresponding jute fiber/PP composites (b) were shown in Figure 7. It clearly indicated that the jute fiber pull-out was quite higher and the bonding between jute and PP was not so good. Small gaps were evident in the matrix near the jute fibers. This was reflected in the IFSS values described in the previous section. SEM images of bamboo fiber (a) and the fracture sides of the corresponding bamboo fiber/PP composites (b) were shown in Figure 8. The SEM image of the fracture surface explained that the fiber pull-out is quite low and fractures between bamboo fiber and PP matrix were clearly evident which indicated excellent fiber matrix adhesion. Also, very few holes in the matrix were reported, suggesting very good bonding between bamboo fiber and the polymer matrix. The IFSS values reported above supported these SEM images. From the SEM images of the fracture surfaces of both jute fiber/PP and bamboo fiber/PP composite, a clear pictorial view was evident and this revealed the interfacial properties of the composite as well as explained why jute-based composites had low mechanical and interfacial properties compared to the bamboo-based composites. From the comparative studies of the mechanical properties between jute fiber/PP and bamboo fiber/PP composites, it was found that 
(a)

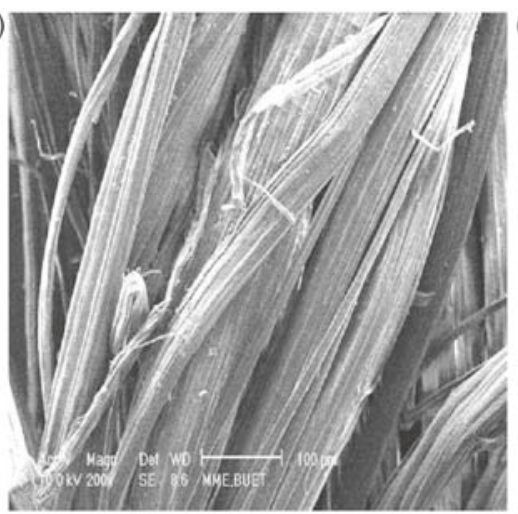

(b)

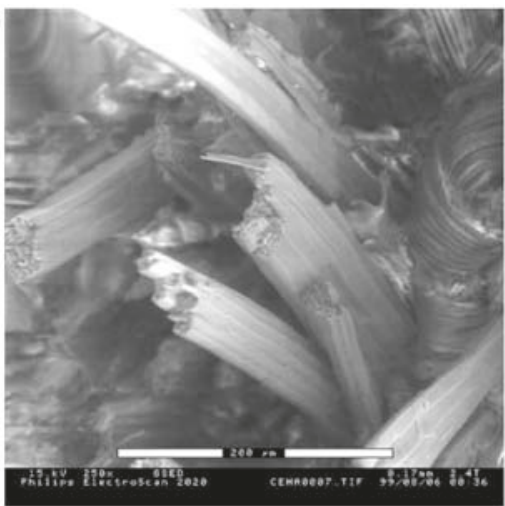

Figure 7. SEM images of (a) jute fiber and (b) fracture surface of jute fiber/PP composite.

(a)
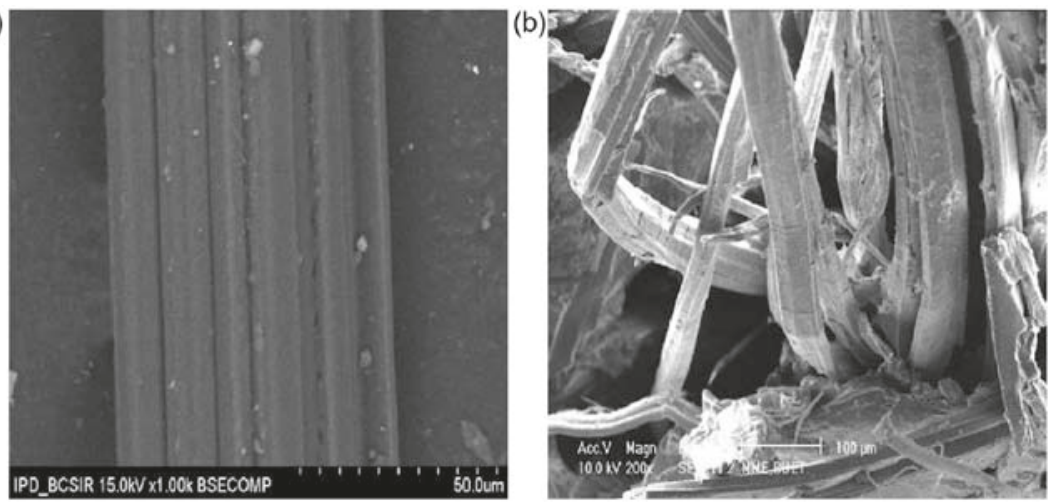

Figure 8. SEM images of (a) bamboo fiber and (b) fracture surface of bamboo fiber/PP composite.

the mechanical properties of jute composites were quite low compared to that of the bamboo-based composite. The IFSS of the composites also revealed the same results.

\section{CONCLUSIONS}

Water uptake of jute and bamboo fibers was studied and it was found that jute fiber gained higher amount of water within a very short time compared 
to the bamboo fiber. Jute fiber-reinforced PP-based composites ( $50 \%$ fiber by weight) were fabricated by compression molding, and the mechanical properties evaluated. TS, BS, TM, and BM of the composites were found to be 48, 56, 900, and $1500 \mathrm{MPa}$, respectively. Then, bamboo fiber-reinforced PP-based composites (50\% fiber by weight) were fabricated and the mechanical properties were investigated. For bamboo-based composites, $\mathrm{TS}$, BS, TM, and $\mathrm{BM}$ were found to be $60,76,4210$, and $6210 \mathrm{MPa}$, respectively. From this investigation, it was found that bamboo fiber/PP composite showed almost $25 \%$ and $35 \%$ improvements in TS and BS over the jute composites. It was also revealed that TM and BM improved by $367 \%$ and $314 \%$, respectively, than that of the jute/PP composites. Degradation tests of the composite in soil medium showed that, bamboo fiber/PP composites retained much of their mechanical properties compared to the jute fiber/PP composite. The IFSS values of both types of composite were measured by SFFT and those of the bamboo and jute-based composites were found to be 4.91 and $2.14 \mathrm{MPa}$, respectively. SEM images of the fracture sides of the composite supported the fact that jute fiber-based PP composite had poorer fiber matrix adhesion than the bamboo fiber-based PP composite.

\section{REFERENCES}

1. Saheb, D.N. and Jog, J.P. (1999). Natural Fiber Polymer Composites: A Review, Advances in Polymer Technology, 18(4): 351-363.

2. Khan, R.A., Khan, M.A., Zaman, H.U., Khan, M.N. and Sultana, S. (2010). Comparative Studies of Mechanical and Interfacial Properties Between Jute and E-glass Fibers Reinforced Polypropylene Composites, Journal of Reinforced Plastics and Composites, 29(7): 1078-1088.

3. Khan, M.A., Hossain, M. and Ali, K.M.I. (1999). Jute Composite with MMA by Gamma and UV Radiations in the Presence of Additives, Journal of Applied Polymer Science, 74: 900-906.

4. Zaman, H., Khan, R.A., Khan, M.A., Khan, A.H. and Hossain, M.A. (2009). Effect of Gamma Radiation on the Performance of Jute Fabrics Reinforced Polypropylene Composites, Radiation Physics and Chemistry, 78: 986-993.

5. Khan, M.A., Ali, K.M.I. and Akhunzada, K.S. (1999). In-situ Grafting of Jute Yarn with HEMA by UV Radiation, Journal of Applied Polymer Science, 77: 841-848.

6. Khan, M.A., Ali, M.A., Ali, K.M.I. and Hinrichsen, G. (1998). Mechanical Studies of MMA Treated Jute and Flax Fibers under UV Radiations, Journal of Applied Polymer Science, 70: 843-850.

7. Khan, M.A. and Ali, K.M.I. (1999). Effect of Titanium Complex in the Modification of Cotton with Urethane Acrylate, Advanced Polymer Technology, 18(3): 267-275.

8. Khan, M.A., Hassan, M.M. and Drazal, L.T. (2005). Effect of 2-Hydroxyethyl Methacrlyate (HEMA) on the Mechanical and Thermal Properties of Jute/Polycarbonate Composites, Composites Part A: Applied Science and Manufacturing, 36: 71-81. 
9. Mohanti, A.K., Khan, M.A. and Hinrichsen, G. (2000). Surface Modification of Jute and its Influence on Performance of Biodegradable Jute-Fabric/Biopol Composites, Composites Science and Technology, 60: 1115-1124.

10. Thwea, M.M. and Liaob, K. (2003). Durability of Bamboo-glass Fiber Reinforced Polymer Matrix Hybrid Composites, Composites Science and Technology, 63: 375-387.

11. Deshpande, A.P., Rao, M.B. and Rao, L. (2000). Extraction of Bamboo Fibers and Their Use as Reinforcement in Polymeric Composites, Journal of Applied Polymer Science, 76: $83-92$.

12. Xiao Chen, X., Guo, Q. and Mi, Y. (1998). Bamboo Fiber-Reinforced Polypropylene Composites: A Study of the Mechanical Properties, Journal of Applied Polymer Science, 69: 1891-1899.

13. Khan, R.A., Khan, M.A., Sultana, S., Nuruzzaman Khan, M., Shubhra, Q.T.H. and Noor, F.G. (2010). Mechanical, Degradation and Interfacial Properties of Synthetic Degradable Fiber Reinforced Polypropylene Composites, Journal of Reinforced Plastics and Composites, 29(3): 466-476.

14. Khan, R.A. Khan, A., Huq, T., Noor, N. and Khan, M.A. (2010). Studies on the Mechanical, Degradation and Interfacial Properties of Calcium Alginate Fiber Reinforced PP Composites, Journal of Polymer Plastics Technology and Engineering, 49: 407-413.

15. Rana, A.K., Mandal, A., Mitra, B.C., Jacobson, R., Rowell, R. and Banerjee, A.N. (1998). Short Jute Fiber-Reinforced Polypropylene Composites: Effect of Compatibilizer, Journal of Applied Polymer Science, 69: 329-338.

16. Cabral, F.S., Paiva, M.C., Nunes, J.P. and Bernardo, C.A. (2003). A Novel Technique for the Interfacial Characterization of Glass Fiber-Polypropylene Systems, Polymer Testing, 22(8): 907-913.

17. Mishra, S., Mohanty, A.K., Drzal, L.T., Misra, M., Parija, S., Nayak, S.K. and Tripathy, S.S. (2003). Studies on Mechanical Performance of Biofibre/Glass Reinforced Polyester Hybrid Composites, Composite Science and Technology, 63: 1377-1385.

18. Greenfield, M.J., Pedicini, A. and Penn, L.S. (2000). Development of a Single Fiber Fragmentation Test for High Strain Rates, International Journal of Adhesion Adhesives, 20(5): 403-407.

19. Kelly, A. and Tyson, W.R. (1965). Tensile Properties of Fibre Reinforced Metals: Copper/Tungsten, Copper/Molybdenum, Journal of the Mechanics and Physics of Solids, 13: $329-350$.

20. Drzal, L.T., Rich, M.J., Koeng, M.F. and Lloyd, P.F. (1983). Adhesion of Graphite Fibres to Epoxy Matrices, The Journal of Adhesion, 16(2): 133-152.

21. Wu, H.F. and Netravali, A.N. (1992). Weibull Analysis of Strength-Length Relationships in Single Nickel on SiC Fibres, Journal of Materials Science, 27(12): 3318-3324.

22. Zhou, X.F., Wagner, H.D. and Nutt, S.R. (2001). Interfacial Properties of Polymer Composites Measured by Push-Out and Fragmentation Tests, Composites Part A: Applied Science and Manufacturing, 32(11): 1543-1551.

23. Slivka, M.A., Chu, C.C. and Adisaputro, I.A. (1997). Fiber-Matrix Interface Studies on Bioabsorbable Composite Materials for Internal Fixation of Bone Fractures. I. Raw Material Evaluation and Measurement of Fiber-Matrix Interfacial Adhesion, Journal of Biomedical Materials Research, 36(4): 469-477.

24. Khan, M.A., Shehrzade, S. and Hassan, M.M. (2004). Effect of Alkali and Ultraviolet (UV) Radiation Pretreatment on Physical and Mechanical Properties of 1,6-Hexanediol Diacrylate-Grafted Jute Yarn by UV Radiation, Journal of Applied Polymer Science, 92(1): $18-24$. 
25. Amada, S., Ichikawa, Y., Munekata, T. Nagase, Y. and Shimizu, K. (1997). Fiber Texture and Mechanical Graded Structure of Bamboo, Composites Part B: Engineering, 28(B): 13-20.

26. Lakkad, S.C. and Patel, J.M. (1980). Mechanical Properties of Bamboo, a Natural Composite, Fiber Science and Technology, 14: 319-322.

27. Khan, R.A., Parsons, A.J., Jones, I.A., Walker, G.S. and Rudd, C.D. (2009). Surface Treatment of Phosphate Glass Fibers using 2-Hydroxyethyl Methacrylate: Fabrication of Poly(Caprolactone)-based Composites, Journal of Applied Polymer Science, 111(1): 246-254.

28. Khan, M.A., Khan, R.A., Ghoshal, S. and Saha, M. (2009). Study on the Physico-Mechanical Properties of Starch-Treated Jute Yarn-Reinforced Polypropylene Composites: Effect of Gamma Radiation, Polymer Plastic Technology and Engineering, 48(5): $542-548$.

29. Hassan, M.M., Islam, M.R. and Khan, M.A. (2003). Improvement of the PhysicoMechanical Properties of Jute Yarn by Photo-grafting with 3-Trimethoxysisyl Propylmethacrylate, Journal of Adhesion Science and Technology, 17(5): 737-750. 